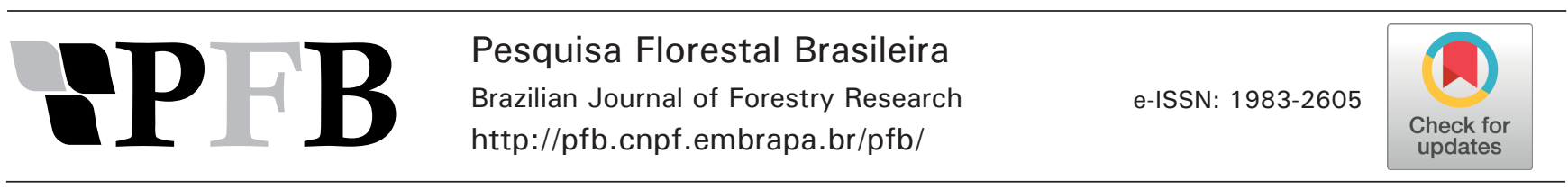

\title{
Biossólido e substrato comercial na produção de mudas de Schinus terebinthifolia
}

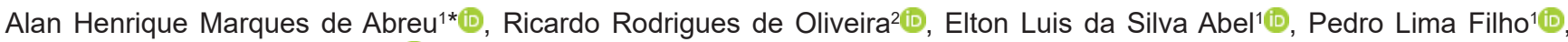 \\ Paulo Sérgio dos Santos Leles ${ }^{1}$ (D)
}

${ }^{1}$ Companhia Estadual de Águas e Esgotos do Rio de Janeiro, BR 465, Km 19,5, Prados Verdes, CEP 26298-566 Nova Iguaçu, RJ, Brasil

2Universidade Federal Rural do Rio de Janeiro, BR 465, Km 47, CEP 23890-000, Seropédica, RJ, Brasil

"Autor correspondente:
alanhenriquem@gmail.com

Termos para indexação:

Lodo de esgoto

Adubação de cobertura

Aroeira pimenteira

Index terms:

Sewage sludge

Top-dressing

Aroeira pepper

Histórico do artigo:

Recebido em 02/10/2015

Aprovado em 21/06/2018

Publicado em 11/12/2018

doi: 10.4336/2018.pfb.38e201501066

\begin{abstract}
Resumo - Atualmente a aquisição de substratos em quantidade e qualidade para a produção de mudas florestais é um grande desafio para o setor florestal, sendo a busca por substratos sustentáveis uma tendência ecológica, econômica e social. O objetivo deste trabalho foi comparar o crescimento, em viveiro e no primeiro ano após o plantio, de mudas de Schinus terebinthifolia Raddi (aroeira pimenteira) produzidas com biossólido (BIO) e com substrato comercial (SC), sob doses de monoamônio fosfato (MAP) em adubação de cobertura. O MAP foi aplicado em doses crescentes nos tratamentos, a cada 21 dias em adubação de cobertura. O BIO, mesmo sem fertilização mineral complementar, favoreceu o crescimento em viveiro, resultando em mudas de qualidade morfológica superior às produzidas em SC sob adubação de cobertura. Tanto a utilização de BIO, quanto o uso de adubação de cobertura em níveis mais elevados podem ser utilizados para aumentar o estabelecimento e o crescimento das mudas de aroeira pimenteira em campo.
\end{abstract}

\section{Biosolids and commercial substrate for production of Schinus terebinthifolia seedlings}

\begin{abstract}
Currently to adquire substrates in quantity and quality for forest seedlings production is a great challenge for the forest sector. The search for sustainable substrates is an ecological, economic and social trend. The objective of this work was to compare seedlings of Schinus terebinthifolia (aroeira pepper) produced with biosolids (BIO) and with commercial substrate (CS), under monoammonium phosphate (MAP) fertilization, in the nursery and in the first year after planting. The MAP was applied as cover fertilization in increasing doses in the treatments, every 21 days. Even without supplement of mineral fertilization, BIO favored seedlings growth in nursery, resulting in seedlings of superior morphological quality when compared to those produced in $\mathrm{CS}$ under cover fertilization. BIO and cover fertilization at higher levels can be used to increase the establishment and growth of aroeira pepper seedlings in the field.
\end{abstract}

\section{Introdução}

A produção de mudas florestais nativas em quantidade e com qualidade é uma das fases mais importantes para o sucesso da recomposição florestal, com grande influência na sobrevivência e crescimento inicial em campo (Abreu et al., 2015; Melo et al., 2018). Dentre os fatores que contribuem para a formação de mudas florestais de qualidade, pode-se destacar tanto o substrato utilizado, como a fertilização química empregada (Caldeira et al., 2013a; Cabreira et al., 2017). 
A escolha de substratos formados por resíduos pode ser uma alternativa viável para a produção de mudas florestais em detrimento aos substratos comerciais disponíveis no mercado, o que pode minimizar os custos de produção dos viveiristas (Abreu et al., 2017). Dentre os resíduos urbanos com maior potencial para utilização como substrato para produção de mudas florestais temse o biossólido resultante de lodo de esgoto estabilizado (Santos et al., 2014). Os altos teores de nutrientes e matéria orgânica presentes neste resíduo podem promover significativo aumento na qualidade das mudas produzidas, além de economia de fertilizantes químicos (Trigueiro \& Guerrini, 2014). A utilização de biossólido como substrato na produção de mudas florestais surge como uma alternativa factível, visto que, em geral, é um material disponível em grandes quantidades, baixo custo e possui elevado valor nutricional (Caldeira et al., 2012). Desta forma, o uso de biossólidos na produção de mudas florestais pode significar não apenas uma alternativa de interesse ambiental, como também de relevante interesse econômico, trazendo vantagem tanto para os geradores do resíduo, quanto para os viveiristas.

Para testar a efetividade de novos substratos na produção de mudas florestais é importante avaliar não apenas a qualidade das mudas produzidas, como também o crescimento após o plantio em campo. Devese, ainda, priorizar os estudos de espécies de grande valor ambiental e que sejam amplamente utilizadas em plantios de recomposição florestal. A aroeira pimenteira (Schinus terebinthifolia Raddi) pertence à família Anacardiaceae. Segundo Lorenzi (2002), em geral, é uma espécie de pequeno porte, rápido crescimento e ciclo de vida relativamente curto, além de ser heliófila e pioneira. Geralmente, os indivíduos dessa espécie colonizam áreas abertas, especialmente margens de rios e terrenos aluviais, possuindo grande atratividade para a fauna (Martins, 2001).

O objetivo deste trabalho foi comparar o crescimento em viveiro e no primeiro ano após o plantio, de mudas de Schinus terebinthifolia (aroeira pimenteira) produzidas com biossólido e com substrato comercial, sob doses de monoamônio fosfato em adubação de cobertura.

\section{Material e métodos}

\section{Fase de produção de mudas}

A produção de mudas foi conduzida no viveiro florestal do Instituto de Florestas da Universidade
Federal Rural do Rio de Janeiro, no período de setembro de 2012 a fevereiro de 2013, em Seropédica, RJ, região da baixada fluminense $\left(22^{\circ} 45^{\prime} 27^{\prime \prime} \mathrm{S}\right.$; $43^{\circ} 41^{\prime} 46^{\prime \prime} \mathrm{W}$; $30 \mathrm{~m}$ ). O clima da região, segundo a classificação de Köppen, é do tipo Aw (Brasil, 1980), tropical com chuvas de verão. Durante o período de produção, a temperatura mínima foi de $19{ }^{\circ} \mathrm{C}$ (alguns dias do mês de setembro) e a máxima entre $36{ }^{\circ} \mathrm{C}-39{ }^{\circ} \mathrm{C}$ (nos meses de dezembro e janeiro).

O delineamento utilizado foi o inteiramente casualizado, com sete tratamentos e quatro repetições, sendo cada unidade experimental formada por 12 mudas. Os tratamentos testados foram: $\mathrm{T}_{1}$ : biossólido sem adubação (BIO+0,00); e $\mathrm{T}_{2}$ : biossólido mais 0,05 $\mathrm{g}$ de monoamônio fosfato amônio (MAP) por muda e por adubação, aplicado em adubação de cobertura a cada 21 dias $(\mathrm{BIO}+0,05)$. Os demais tratamentos foram com substrato comercial ( $\mathrm{SC}$ - $\mathrm{T}_{3}$ : $\mathrm{SC}$ sem adubação $(\mathrm{SC}+0,00) ; \mathrm{T}_{4}: \mathrm{SC}$ mais $0,05 \mathrm{~g} \mathrm{de} \mathrm{MAP} \mathrm{muda}^{-1}(\mathrm{SC}+0,05)$; $\mathrm{T}_{5}$ : SC mais $0,10 \mathrm{~g}$ de MAP muda ${ }^{-1}(\mathrm{SC}+0,10) ; \mathrm{T}_{6}: \mathrm{SC}$ mais 0,20 g de MAP muda $^{-1}(\mathrm{SC}+0,20) ; \mathrm{T}_{7}: \mathrm{SC}$ mais 0,40 $\mathrm{g}$ de MAP muda $^{-1}(\mathrm{SC}+0,40)$, aplicados em adubação de cobertura. Foram realizadas cinco adubações de cobertura em intervalos de 21 dias, sendo a primeira aos 50 e a última aos 134 dias após a semeadura. A fertilização de cobertura foi realizada via solução aquosa, aplicada com o auxílio de seringa. Em cada tubete, foram aplicados $10 \mathrm{~mL}$ de solução contendo doses de MAP correspondentes aos tratamentos.

O biossólido utilizado foi disponibilizado pela Companhia Estadual de Águas e Esgoto do Rio de Janeiro (CEDAE), sendo oriundo da Estação de Tratamento de Esgotos (ETE) Alegria, localizada no bairro Caju, Rio de Janeiro, RJ. O esgoto tratado pela ETE Alegria é derivado de áreas urbanas domiciliares e comerciais e tratado por processo de digestão anaeróbica.

O biossólido foi caracterizado de acordo com as exigências da resolução CONAMA nº 375/2006 (Brasil, 2006). Os resultados das análises (Tabela 1), atenderam aos parâmetros relativos à concentração dos agentes patogênicos e indicadores bacteriológicos, sendo classificado como de classe $\mathrm{A}$, e aos relativos à presença de compostos inorgânicos, apresentando valores inferiores aos máximos estipulados pela legislação, sendo considerado apto para uso em ambientes agrícolas.

Para a formulação dos substratos, o biossólido da ETE Alegria, que primeiramente passa pelo processo de secagem natural como método de redução de patógenos, foi peneirado com auxílio de peneiras de malha de $10 \mathrm{~mm}$. 
Tabela 1. Concentração de microrganismos patogênicos e substâncias inorgânicas $\left(\mathrm{mg} \mathrm{kg}^{-1}\right)$ potencialmente tóxicas no biossólido proveniente da Estação de Tratamento de Esgotos (ETE) Alegria, Rio de Janeiro, RJ, e os valores máximos permitido pela Resolução CONAMA nº 375/2006 (Brasil, 2006).

Table 1. Concentration of pathogenic microorganisms and potentially toxic inorganic substances $\left(\mathrm{mg} \mathrm{kg}^{-1}\right)$ in the biosolid from Alegria Sewage Treatment Station (ETE), Rio de Janeiro, $\mathrm{RJ}$, and the maximum values acceptable by CONAMA Resolution 375/2006 (Brazil, 2006).

\begin{tabular}{ccc}
\hline Parâmetro & ETE Alegria $^{2}$ & CONAMA $^{1}$ \\
\hline $\begin{array}{c}\text { Coliformes termotolerantes } \\
\text { (NMP g-1 ST) }\end{array}$ & $<0,04$ & $<1000$ \\
$\begin{array}{c}\text { Ovos viáveis de helmintos (Ovos } \\
\left.\mathrm{g}^{-1} \mathrm{ST}\right)\end{array}$ & $<0,01$ & $<0,25$ \\
$\begin{array}{c}\text { Salmonella } \mathrm{sp} \text {. (Presente/ausente } \\
\text { em 10g ST) }\end{array}$ & Ausente & Ausente \\
$\mathrm{As}$ & 2,6 & 41 \\
$\mathrm{Ba}$ & 157 & 1300 \\
$\mathrm{Cd}$ & 0,2 & 39 \\
$\mathrm{~Pb}$ & 197 & 300 \\
$\mathrm{Cu}$ & 267 & 1500 \\
$\mathrm{Cr}$ & 70 & 1000 \\
$\mathrm{Hg}$ & 0,03 & 17 \\
$\mathrm{Mo}$ & 22,6 & 500 \\
$\mathrm{Ni}$ & 40,2 & 420 \\
$\mathrm{Zn}$ & 5,9 & 100 \\
\hline
\end{tabular}

$\mathrm{NMP}=$ número mais provável; ST = sólidos totais. ${ }^{1}$ Fonte: Brasil (2006).

O substrato comercial utilizado na formulação dos tratamentos foi o Mecplant ${ }^{\circledR}$ Florestal 3, indicado para produção de mudas de espécies nativas propagadas por sementes. Este substrato é amplamente utilizado na produção de mudas florestais em tubetes e, de acordo com o fabricante, é um material produzido, integralmente, a partir da casca de Pinus sp. bioestabilizada. Os dois substratos foram submetidos à análise química, realizada pelo Laboratório de Análise de Solos Viçosa Ltda (Tabela 2).

As mudas de aroeira pimenteira foram produzidas em tubetes, com capacidade volumétrica de $280 \mathrm{~cm}^{3}$, dispostos em canteiros suspensos e localizados a pleno sol. A semeadura foi realizada diretamente nos recipientes, distribuindo-se três sementes por recipiente. A irrigação foi realizada por sistema de microaspersão de uma a três vezes ao dia, dependendo das condições do clima (chuva, insolação e temperatura) e da fase de crescimento das mudas. Foi mantido sobre os canteiros um sombrite com $50 \%$ de interceptação de luminosidade, até os 20 dias após a semeadura, quando foi realizado o desbaste, deixando-se apenas uma plântula por recipiente, utilizando-se como critério a plântula de maior vigor e mais centralizada.

Tabela 2. Análise química do biossólido (BIO) proveniente da Estação de Tratamento de Esgotos Alegria e do substrato comercial (SC).

Table 2. Chemical analysis of the biosolid (BIO) from Alegria Sewage Treatment Station and of the commercial substrate (SC).

\begin{tabular}{|c|c|c|c|c|c|c|c|c|c|}
\hline \multirow[t]{2}{*}{ Substrato } & $\mathbf{N}$ & $\mathbf{P}$ & $\mathbf{K}$ & $\mathrm{Ca}$ & Mg & $\mathbf{S}$ & $\mathrm{CO}$ & $\mathrm{C} / \mathrm{N}$ & $\mathrm{pH}$ \\
\hline & & & & - - (\% & & 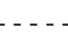 & - - - & - & - \\
\hline $\mathrm{SC}$ & 0,49 & 0,17 & 0,16 & 1,49 & 0,58 & 0,31 & 11,85 & 24 & 4,2 \\
\hline $\mathrm{BIO}$ & 1,42 & 0,55 & 0,40 & 1,85 & 0,58 & 1,31 & 8,89 & 7 & 5,8 \\
\hline
\end{tabular}

Teores totais determinados no extrato ácido (ácido nítrico com ácido perclórico); $\mathrm{N}$ - determinado pelo método do Kjeldahl; $\mathrm{CO}=$ carbono orgânico - determinado pelo método Walkley - Black.

Aos 50 dias após a semeadura mediu-se a altura $(\mathrm{H})$ das mudas, com auxilio de uma régua graduada. $\mathrm{O}$ diâmetro do coleto (D) foi mensurado pela primeira vez aos 71 dias, com paquímetro digital. As duas variáveis continuaram sendo mensuradas a cada 21 dias até aos 155 dias após a semeadura. Após a última avaliação de altura e diâmetro, selecionaram-se as cinco mudas mais próximas da média de cada repetição para determinação da matéria seca da parte aérea (MSPA) e a matéria seca radicular (MSR). As mudas selecionadas tiveram o substrato lavado em água corrente com auxilio de uma peneira, para evitar perda de raízes. O sistema radicular e a parte aérea das mudas foram acondicionados em sacos de papel e levados à estufa de circulação de ar forçada por $72 \mathrm{~h}$, quando atingiram peso constante.

Com os dados dos parâmetros obtidos aos 155 dias após a semeadura, foram calculados os parâmetros de qualidade: relação entre altura e diâmetro (H/D), relação entre a matéria seca da parte aérea e radicular (MSPA/ MSR) e o índice de qualidade de Dickson (equação 1) (Dickson et al., 1960). 


$$
\mathrm{IQD}=\frac{M S T}{(\mathrm{H} / \mathrm{D})+(M S P A / M S R)}
$$

Em que, IQD = índice de qualidade de Dickson; MST = matéria seca total; MSPA = matéria seca da parte aérea; $\mathrm{MSR}=$ matéria seca do sistema radicular; $\mathrm{H}=$ altura da parte aérea; $\mathrm{D}=$ diâmetro de coleto.

\section{Fase de campo}

Para avaliação da sobrevivência e crescimento inicial em campo foram implantadas 20 mudas de cada tratamento, sendo cinco de cada repetição. Foram excluídas da implantação de campo as mudas referentes aos tratamentos que obtiveram, ao final do ciclo de produção, médias inferiores às adotadas como padrão de qualidade para expedição de mudas desta espécie (altura $\geq 25 \mathrm{~cm}$; diâmetro do coleto $\geq 3,00 \mathrm{~mm}$ ), estipulados por José et al. (2005).

O experimento foi implantado em 28 de fevereiro de 2013, em área de propriedade da Pequena Central Hidrelétrica (PCH) Santa Rosa, no município de Bom Jardim, localizado na região Centro Fluminense do Estado do Rio de Janeiro. A classe de solo do local é Latossolo Vermelho Amarelo Distrófico. Segundo a classificação de Köppen, o clima na região é classificado como Aw - tropical chuvoso, com inverno seco e verão chuvoso. A altitude no local é de $530 \mathrm{~m}$ e a precipitação média anual é de $1.402 \mathrm{~mm}$, com período seco compreendido entre os meses de junho a setembro e o período chuvoso de novembro a março. A temperatura média anual da região é de $22{ }^{\circ} \mathrm{C}$, com temperatura média mínima em torno de $16{ }^{\circ} \mathrm{C}$, no mês de julho, e média máxima de $28^{\circ} \mathrm{C}$, no mês de fevereiro.

$\mathrm{O}$ delineamento experimental foi inteiramente casualizado, sendo testados cinco tratamentos em campo, com 20 repetições por tratamento, cada planta representando uma parcela. Realizou-se a marcação das covas, o coroamento e a abertura das covas com dimensões de $25 \mathrm{~cm} \times 25 \mathrm{~cm}$ x $25 \mathrm{~cm}$, num espaçamento de 3,2 $\mathrm{m}$ x 1,7 m. Em seguida, foi realizada a adubação com $120 \mathrm{~g}$ de superfosfato simples por cova e o plantio das mudas. Foi realizado o controle de plantas daninhas em três ocasiões, utilizando-se a capina em faixa e roçada da entrelinha. Realizou-se também, durante todo o período, o controle de formigas cortadeiras, através da ronda e distribuição de iscas formicidas.

Foi realizado o acompanhamento quinzenal da precipitação e temperatura máxima (Figura 1), desde o plantio até a data da última avaliação de crescimento, utilizando-se dados da Estação Meteorológica de Cordeiro, RJ (Instituto Nacional de Meteorologia, 2014).

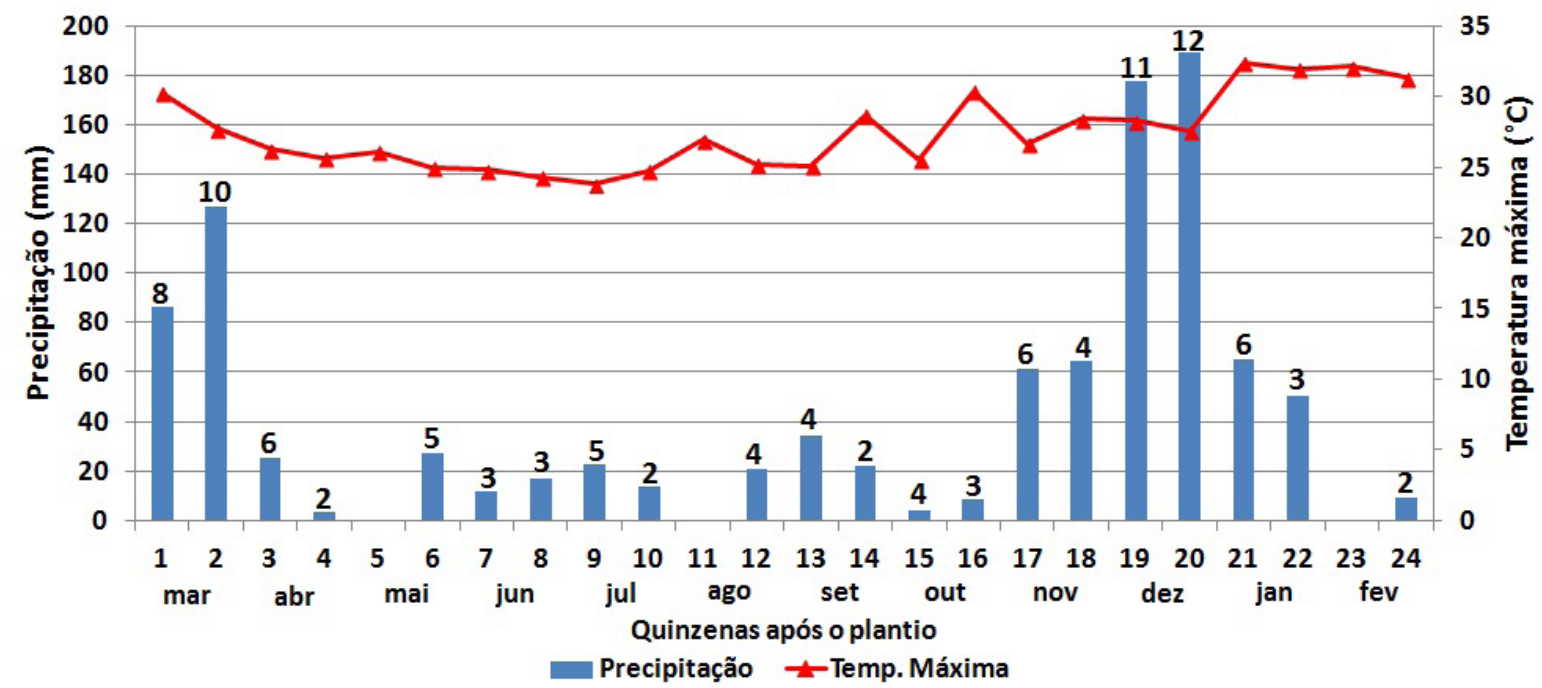

Figura 1. Precipitação e temperatura máximas quinzenais, de março de 2013 a fevereiro de 2014, registrados pela Estação Meteorológica de Cordeiro, município de Cordeiro, RJ. A numeração sobre as barras indica em quantos dias na quinzena houve precipitação. Fonte: Instituto Nacional de Meteorologia (2014).

Figure 1. Maximum precipitation and temperature for every 15 days, from March 2013 to February 2014, recorded by the Meteorological Station of Cordeiro, municipality of Cordeiro, RJ. The numbering on the bars indicates the number of days that there was precipitation in every 15 days period. Source: Instituto Nacional de Meteorologia (2014). 
As avaliações de crescimento em campo consistiram nas medições da altura da parte aérea, com uma régua graduada, e do diâmetro ao nível do solo, com auxílio de um paquímetro digital, no dia do plantio (tempo 0), aos seis e aos 12 meses após o plantio. Além destas medidas, obteve-se também a taxa de sobrevivência aos 12 meses após o plantio, através da contagem das mudas.

Todos os dados, com exceção da sobrevivência, foram submetidos ao teste de Liliefors e de Cochran \& Bartlet, para avaliação da normalidade e homogeneidade das variâncias, respectivamente, sendo que não houve necessidade de transformações. Foi realizada a análise de variância e, posteriormente, o teste de Tukey ao nível de significância de 5\%. As análises foram realizadas com auxílio do programa Sistema de Análise Estatística e Genética - SAEG (Ribeiro Júnior, 2001).

\section{Resultados e discussão}

\section{Fase de produção de mudas}

As mudas produzidas com biossólido na composição do substrato $\left(\mathrm{T}_{1}: \mathrm{BIO}+0,00 ; \mathrm{T}_{2}: \mathrm{BIO}+0,05 \mathrm{~g}\right.$ de MAP muda $^{-1}$ ), apresentaram tendência de maior crescimento em altura e diâmetro do colo durante todo o ciclo de produção em viveiro. Aos 155 dias após a semeadura alcançaram médias significativamente superiores às dos tratamentos com substrato comercial (Figura 2).
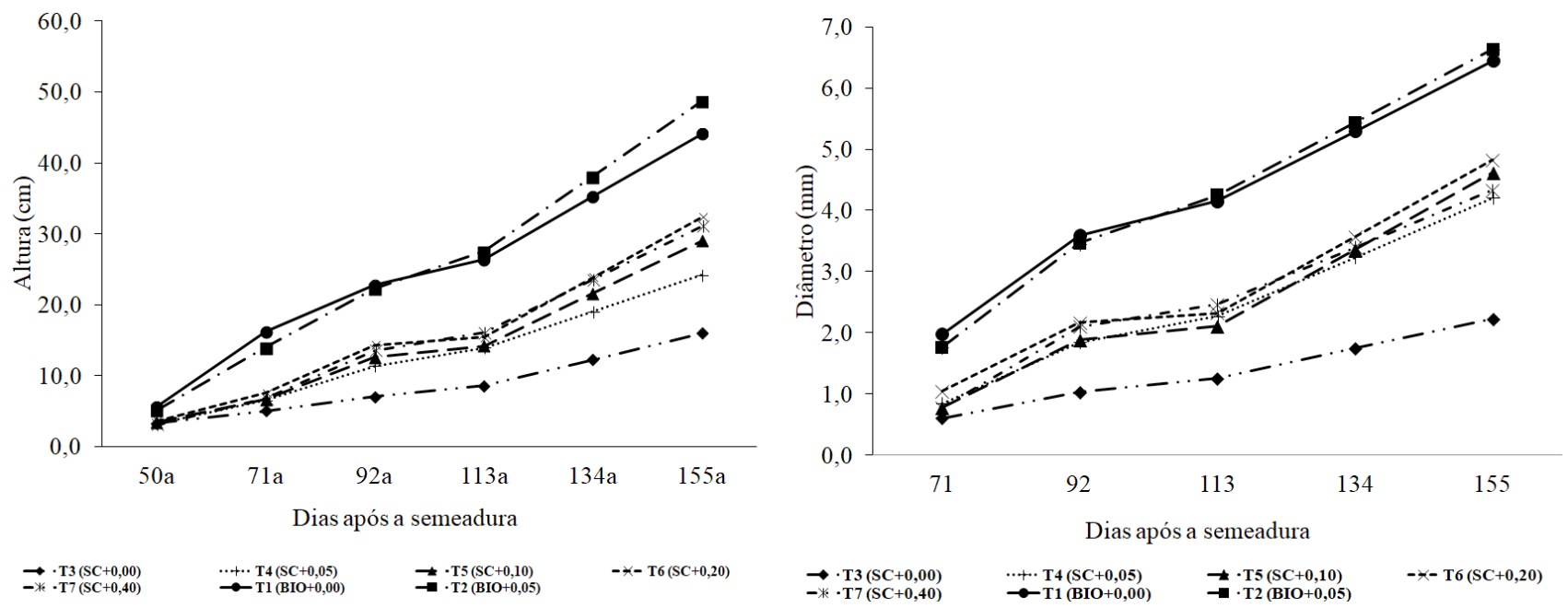

Figura 2. Crescimento ao longo do tempo em altura e diâmetro do coleto, de mudas de Schinus terebinthifolia produzidas em substrato de biossólido (BIO) e substrato comercial (SC) sob diferentes dosagens de monoamônio fosfato (MAP) por adubação. $\mathrm{T}_{1}: \mathrm{BIO}+0,00 ; \mathrm{T}_{2}: \mathrm{BIO}+0,05 \mathrm{~g} \mathrm{de} \mathrm{MAP} \mathrm{muda}^{-1} ; \mathrm{T}_{3}: \mathrm{SC}+0,00 ; \mathrm{T}_{4}: \mathrm{SC}+0,05 \mathrm{~g} \mathrm{de} \mathrm{MAP} \mathrm{muda}^{-1} ; \mathrm{T}_{5}: \mathrm{SC}+0,10 \mathrm{~g}$ de MAP muda $^{-1} ; \mathrm{T}_{6}: \mathrm{SC}+0,20 \mathrm{~g}_{\text {de }} \mathrm{MAP}_{\text {muda }}{ }^{-1} ; \mathrm{T}_{7}: \mathrm{SC}+0,40 \mathrm{~g}$ de MAP muda ${ }^{-1}$. Na última avaliação, para cada característica, valores seguidos da mesma letra não diferem entre si pelo teste de Tukey $(\mathrm{p}<0,05)$.

Figure 2. Height and diameter of Schinus terebinthifolia seedlings grown in biosolid (BIO) and commercial substrate (SC) under different doses of monoammonium phosphate (MAP) by fertilization. $\mathrm{T}_{1}: \mathrm{BIO}+0.00 ; \mathrm{T}_{2}: \mathrm{BIO}+0.05 \mathrm{~g}$ of MAP

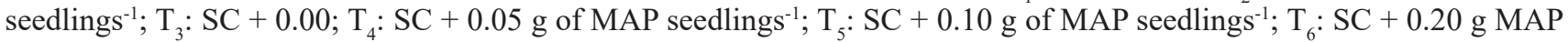
seedlings ${ }^{-1} ; \mathrm{T}_{7}: \mathrm{SC}+0.40 \mathrm{~g}_{\mathrm{O}}$ of MAP seedlings ${ }^{-1}$. In the last evaluation, for each characteristic, values followed by the same letter do not differ by Tukey's test $(\mathrm{p}<0.05)$.

Resultado semelhante ao deste trabalho foi encontrado por Varela \& Martinez (2013), que testando o uso de biossólido em comparação a um substrato tradicional (turfa + pumicita) sob diferentes adubações de cobertura, encontraram as maiores médias de altura e diâmetro de Nothofagus alpina nos tratamentos com biossólido. Caldeira et al. (2013b) também encontraram maior crescimento em altura e diâmetro de mudas de eucalipto produzidas em substratos com lodo de esgoto e vermiculita, do que em substrato comercial à base de casca de pinus bioestabilizada. Os autores atribuíram esse maior crescimento à maior disponibilidade dos nutrientes contidos no biossólido, o que melhora a nutrição das mudas e, consequentemente, acelera o crescimento vegetativo. 
José et al. (2005) estipularam que mudas de aroeira pimenteira com adequada qualidade para expedição para campo devem apresentar no mínimo $25 \mathrm{~cm}$ de altura e $3 \mathrm{~mm}$ de diâmetro do coleto. Constata-se que as mudas produzidas com biossólido atingiram esse padrão mínimo aproximadamente aos 100 dias após a semeadura. No entanto, nos tratamentos com substrato comercial esse padrão foi alcançado aproximadamente aos 140 dias. Vale destacar que no Estado do Rio de Janeiro o padrão exigido para plantio em recomposição florestal é de no mínimo $40 \mathrm{~cm}$ de altura. Por esse motivo, o experimento foi mantido até que pelo menos um dos tratamentos atingisse esse padrão.

Pode-se inferir que o uso de biossólido na composição do substrato, atrelado ao manejo de rustificação adequado no viveiro, pode trazer vantagens relacionadas ao crescimento das mudas de aroeira pimenteira, como também na redução do ciclo de produção, resultando em aumento da produtividade dos viveiros com mudas de qualidade. A eficiência do biossólido para o encurtamento do ciclo de produção de mudas também foi observada por Basil et al. (2009) e Namiot et al. (2012) para Austrocedrus chilensis e por Varela \& Martinez (2013) para Nothofagus alpina.

A semelhança em altura e diâmetro do coleto entre as mudas dos tratamentos $\mathrm{T}_{1}$ e $\mathrm{T}_{2}$, que continham biossólido como substrato, possivelmente é decorrente da disponibilidade de nutrientes e matéria orgânica contida neste material, que supriram de forma eficiente a demanda das mudas de aroeira pimenteira durante todo o ciclo, mesmo no tratamento sem adubação. Como os nutrientes presentes na composição do biossólido se encontram em grande parte na forma orgânica, a liberação gradativa pode ter contribuído para a maior eficiência de utilização dos nutrientes pelas mudas, refletindo em maior crescimento, quando comparadas com o substrato comercial sob adubação mineral.

Apesar das mudas dos tratamentos $\mathrm{T}_{1}$ e $\mathrm{T}_{2}$ não apresentarem diferenças significativas relacionadas à altura e ao diâmetro do coleto (Figura 2), constata-se pela Tabela 3 que as mesmas diferiram estatisticamente em relação à matéria seca da parte aérea (MSPA). Possivelmente isso é decorrente da maior oferta de $\mathrm{N}$ e $\mathrm{P}$ advindos da adubação de cobertura com MAP no tratamento $T_{2}$, que pode ter contribuído para o crescimento da parte aérea, principalmente das folhas. Segundo Trazzi et al. (2014), teores elevados de N e P são altamente requeridos nos estádios iniciais de desenvolvimento das mudas, atuando no arranque inicial do crescimento da parte aérea.

Tabela 3. Parâmetros de crescimento e índices de qualidade das mudas de Schinus terebinthifolia produzidas em biossólidos (BIO) e substrato comercial (SC) sob diferentes doses de monoamônio fosfato (MAP), aos 155 dias após a semeadura.

Table 3. Growth parameters and quality indexes of Schinus terebinthifolia seedlings produced in biosolids (BIO) and commercial substrate (SC) under different doses of monoammonium phosphate (MAP) at 155 days after sowing..

\begin{tabular}{ccccccc}
\hline & & H/D & MSPA & MSR & $\begin{array}{c}\text { MSPA/ } \\
\text { MSR }\end{array}$ & IQD \\
Trat. & Composição & - & - - - g planta ${ }^{-1}--$ & - & - \\
\hline $\mathrm{T}_{1}$ & $\mathrm{BIO}+0,00$ & $6,84 \mathrm{a}$ & $6,87 \mathrm{~b}$ & $3,68 \mathrm{~b}$ & $1,87 \mathrm{ab}$ & $1,21 \mathrm{a}$ \\
$\mathrm{T}_{2}$ & $\mathrm{BIO}+0,05$ & $7,34 \mathrm{a}$ & $9,57 \mathrm{a}$ & $4,54 \mathrm{a}$ & $2,13 \mathrm{ab}$ & $1,49 \mathrm{a}$ \\
$\mathrm{T}_{3}$ & $\mathrm{SC}+0,00$ & $7,23 \mathrm{a}$ & $0,51 \mathrm{~d}$ & $0,38 \mathrm{~d}$ & $1,38 \mathrm{c}$ & $0,11 \mathrm{c}$ \\
$\mathrm{T}_{4}$ & $\mathrm{SC}+0,05$ & $5,75 \mathrm{a}$ & $2,31 \mathrm{c}$ & $1,44 \mathrm{c}$ & $1,62 \mathrm{bc}$ & $0,52 \mathrm{~b}$ \\
$\mathrm{~T}_{5}$ & $\mathrm{SC}+0,10$ & $6,30 \mathrm{a}$ & $2,88 \mathrm{c}$ & $1,50 \mathrm{c}$ & $1,93 \mathrm{ab}$ & $0,54 \mathrm{~b}$ \\
$\mathrm{~T}_{6}$ & $\mathrm{SC}+0,20$ & $6,71 \mathrm{a}$ & $3,62 \mathrm{c}$ & $1,55 \mathrm{c}$ & $2,32 \mathrm{a}$ & $0,57 \mathrm{~b}$ \\
$\mathrm{~T}_{7}$ & $\mathrm{SC}+0,40$ & $7,26 \mathrm{a}$ & $2,75 \mathrm{c}$ & $1,18 \mathrm{c}$ & $2,29 \mathrm{a}$ & $0,41 \mathrm{~b}$ \\
\hline
\end{tabular}

$\mathrm{T}_{1}: \mathrm{BIO}+0,00 ; \mathrm{T}_{2}: \mathrm{BIO}+0,05 \mathrm{~g}$ de MAP muda $^{-1} ; \mathrm{T}_{3}: \mathrm{SC}+0,00 ; \mathrm{T}_{4}: \mathrm{SC}+0,05$ g de MAP muda ${ }^{-1} ; \mathrm{T}_{5}: \mathrm{SC}+0,10 \mathrm{~g}$ de MAP muda ${ }^{-1} ; \mathrm{T}_{6}: \mathrm{SC}+0,20 \mathrm{~g}$ de MAP muda $^{-1} ; \mathrm{T}_{7}: \mathrm{SC}+0,40 \mathrm{~g}$ de MAP muda ${ }^{-1}$. Médias seguidas da mesma letra, na coluna, não diferem entre si pelo teste de Tukey $(\mathrm{p}<0,05)$.

As mudas produzidas em todos os tratamentos avaliados obtiveram valores de relação H/D abaixo de 10 (Tabela 3), faixa considerada boa, recomendada por Birchler et al. (1998). Mudas com alta relação H/D podem apresentar estiolamento e menor índice de sobrevivência em campo. O tombamento decorrente dessa característica pode resultar também em deformações das plantas.

A matéria seca radicular (MSR) também apresentou diferença significativa entre os dois tratamentos com biossólido, o que pode estar relacionado à maior oferta de P. Provavelmente, o maior suprimento de P oriundo da adubação de cobertura no tratamento $T_{2}$ favoreceu o crescimento do sistema radicular (Carneiro, 1995). Observa-se que mesmo as mudas de aroeira pimenteira produzidas com a maior dose de MAP em substrato comercial (T7) apresentaram crescimento significativamente inferior às mudas produzidas com biossólido sem adubação. 
As mudas de aroeira pimenteira apresentaram valores de relação MSPA/MSR entre 1,38 e 2,32 (Tabela 3). Caldeira et al. (2008) defendem a teoria de que mudas florestais de qualidade devem apresentar valores de MSPA/MSR próximos de 2,00, proporcionando adequada distribuição entre a parte aérea e a radicular. Em geral, a relação MSPA/MSR é menor em ambientes com menor fertilidade, podendo ser considerada estratégia da planta para retirar o máximo de nutrientes naquela condição (Caldeira et al., 2013b). Este fato foi observado nas mudas dos tratamentos $\mathrm{T}_{3}(\mathrm{SC}+0,00) \mathrm{e} \mathrm{T}_{4}$ $(\mathrm{SC}+0,05)$, onde foram observados os menores valores desta relação.

Nóbrega et al. (2007), avaliaram o crescimento de mudas de Schinus terebinthifolia com diferentes doses de terra de subsolo e lodo de esgoto (100:0; 80:20; 60:40; 40:60; e 20:80) sem adubação mineral, e constataram que o lodo de esgoto melhorou a fertilidade do substrato, o que proporcionou aumento na biomassa total. Caldeira et al. (2013a), avaliando diferentes substratos na produção de mudas de Chamaecrista desvauxii, encontraram resultado semelhante, observando que os tratamentos que continham lodo de esgoto em sua composição apresentaram resultados de massa seca da parte aérea, massa seca de raízes e massa seca total estatisticamente superiores às do tratamento com apenas substrato comercial em sua composição.

Outro parâmetro utilizado para avaliação de qualidade de mudas florestais é o índice de qualidade de Dickson (IQD). Quanto maior for esse valor dentro de um lote de mudas, melhor o padrão de qualidade (Delarmelina et al., 2013). Desta forma, observa-se que as mudas produzidas nos tratamentos $\mathrm{T}_{1}$ e $\mathrm{T}_{2}$ apresentaram os maiores valores correspondentes a este índice, sendo o menor valor encontrado em mudas do tratamento $\mathrm{T}_{3}$ (Tabela 3). Trazzi et al. (2014), avaliando o biossólido como substrato para produção de mudas de Tectona grandis, também encontraram nos tratamentos com biossólido valores de IQD superiores aos observados para o substrato comercial. Como o IQD pondera parâmetros importantes empregados para avaliação da qualidade das mudas, pode-se inferir que o biossólido contribuiu para melhorar a qualidade das mudas de aroeira pimenteira, sendo possível produzir mudas de excelente qualidade morfológica, sem a necessidade de adubação de cobertura com MAP (Figura 3).

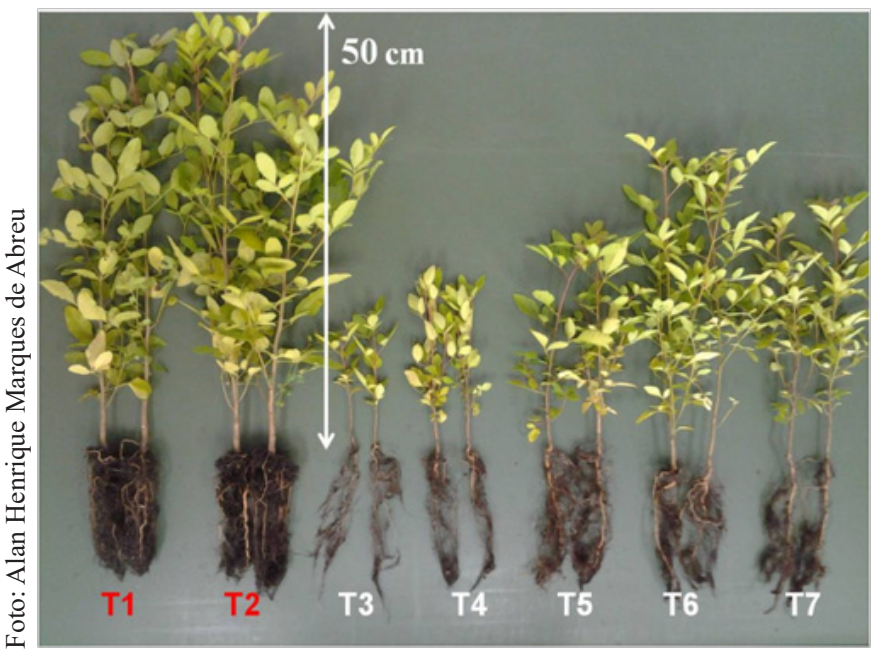

Figura 3. Mudas de aroeira pimenteira (Schinus terebinthifolia) produzidas em biossólido (BIO) e substrato comercial (SC), sob diferentes dosagens de monoamônio fosfato (MAP), aos 155 dias após a semeadura. $\mathrm{T}_{1}: \mathrm{BIO}+0,00 ; \mathrm{T}_{2}: \mathrm{BIO}+0,05 \mathrm{~g}$ de MAP muda ${ }^{-1} ; \mathrm{T}_{3}: \mathrm{SC}+0,00 ; \mathrm{T}_{4}: \mathrm{SC}+0,05 \mathrm{~g}$ de MAP muda 1; $\mathrm{T}_{5}: \mathrm{SC}+0,10 \mathrm{~g}$ de MAP muda $^{-1} ; \mathrm{T}_{6}: \mathrm{SC}+0,20 \mathrm{~g}$ de MAP muda $^{-1} ; \mathrm{T}_{7}: \mathrm{SC}+0,40 \mathrm{~g}^{\mathrm{de}} \mathrm{MAP}$ muda $^{-1}$. Da esquerda para direita corresponde aos denominados $\mathrm{T}_{1}$ a $\mathrm{T}_{7}$.

Figure 3. Seedlings of Schinus terebinthifolia grown in biosolid (BIO) and commercial substrate (SC), under different dosages of monoammonium phosphate (MAP), at 155 days after sowing. $\mathrm{T}_{1}: \mathrm{BIO}+0.00 ; \mathrm{T}_{2}: \mathrm{BIO}+0.05 \mathrm{~g}$ of MAP seedlings $\mathrm{s}^{-1} ; \mathrm{T}_{3}: \mathrm{SC}+0.00 ; \mathrm{T}_{4}: \mathrm{SC}+0.05 \mathrm{~g}$ of MAP seedlings ${ }^{-1}$; $\mathrm{T}_{5}: \mathrm{SC}+0.10 \mathrm{~g}$ of MAP seedlings ${ }^{-1} ; \mathrm{T}_{6}: \mathrm{SC}+0.20 \mathrm{~g}$ MAP seedlings $^{-1} ; \mathrm{T}_{7}: \mathrm{SC}+0.40 \mathrm{~g}$ of MAP seedlings ${ }^{-1}$. $\mathrm{T}_{1}$ to $\mathrm{T}_{7}$ is presented in order from left to right.

Além do maior crescimento relacionado aos parâmetros altura e diâmetro, as mudas produzidas com biossólido apresentaram volume radicular superior às produzidas em substrato comercial (Tabela 3, Figura 3). Apesar das mudas com biossólido responderem à fertilização complementar para algumas características, os nutrientes já presentes foram suficientes para a formação de plantas com boa qualidade morfológica, considerando que na maior parte das características avaliadas não houve diferenças significativas entre os tratamentos $\mathrm{T}_{1}$ e $\mathrm{T}_{2}$

A adubação de cobertura é uma técnica importante para a produção de mudas florestais. No entanto, demanda conhecimento técnico e eleva os custos de produção, tanto pela aquisição de fertilizantes, como pela necessidade de mão-de-obra. A utilização de biossólido 
como substrato pode surgir como uma alternativa para simplificação do processo de produção de mudas em tubetes, eliminando ou reduzindo uma das atividades mais cruciais e emblemáticas deste sistema, que é a necessidade de reposição de nutrientes no substrato.

\section{Fase de campo}

As mudas produzidas nos tratamentos $\mathrm{T}_{3}(\mathrm{SC}+0,00) \mathrm{e}$ $\mathrm{T}_{4}(\mathrm{SC}+0,05)$ apresentaram padrões médios de qualidade em altura e ou diâmetro abaixo dos recomendados por José et al. (2005), aos 155 dias após a semeadura. Portanto, não foram levadas para avaliação em campo.

Todos os demais tratamentos apresentaram sobrevivência das mudas de $100 \%$ aos 12 meses após o plantio em campo, ratificando o padrão de qualidade das mudas produzidas. Segundo Pezzutti \& Caldato (2011), o sucesso da implantação começa com a qualidade morfológica das mudas, que está diretamente associada com a sobrevivência e crescimento inicial em campo.

Além da qualidade das mudas, é indispensável que as condições de implantação e estabelecimento em campo sejam favoráveis. As condições climáticas no primeiro mês após a implantação foi favorável e pode ter contribuído para o sucesso do estabelecimento inicial em campo (Figura 1). Após esta etapa inicial, houve um longo período de estiagem. No entanto, a qualidade das mudas, aliada à rusticidade da espécie e aos tratos culturais e manutenções bem conduzidas, favoreceram a sobrevivência de todas as mudas de aroeira pimenteira em todos os tratamentos, mesmo enfrentando um período com baixa precipitação.

Devido à sua rusticidade, a aroeira pimenteira é amplamente recomendada para projetos de reflorestamento, na restauração de matas ciliares, recuperação de solos pouco férteis (rochosos, salino hidromórficos), além da recuperação de áreas degradadas (Carvalho, 2003; José et al., 2005). A capacidade de adaptação a diferentes ambientes é um dos fatores que podem ter contribuído para a sobrevivência de $100 \%$ das mudas em todos os tratamentos.

Segundo a definição de Johnson \& Cline (1991), mudas de alta qualidade são aquelas produzidas a baixo custo, que podem se adequar aos atuais sistemas de plantio, além de sobreviverem e crescerem bem após o plantio. Portanto, para definir se as mudas implantadas em campo possuem de fato qualidade desejável não basta avaliar apenas a sobrevivência, mas também o crescimento após o plantio. Observa-se pela Figura 4 que as mudas produzidas nos tratamentos $\mathrm{T}_{6}(\mathrm{SC}+0,20)$ e $\mathrm{T}_{7}(\mathrm{SC}+0,40)$, que apresentavam no momento da

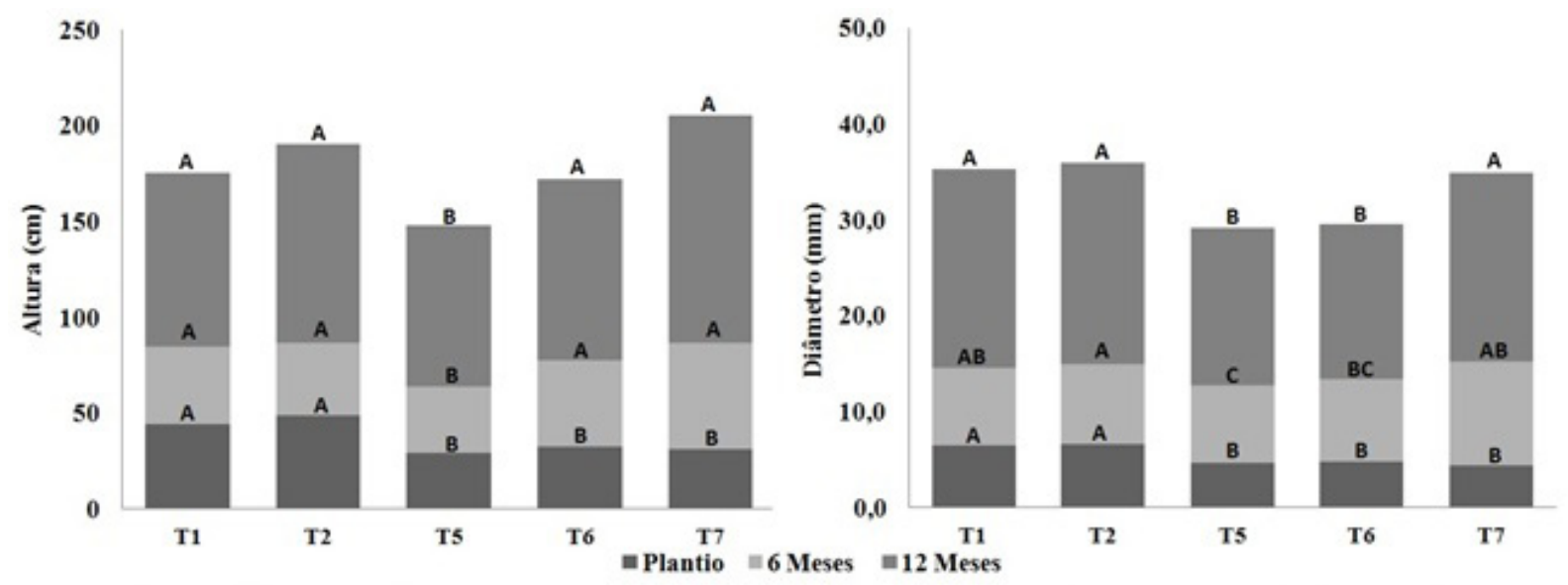

Figura 4. Altura e diâmetro de coleto de aroeira pimenteira (Schinus terebinthifolia) no plantio, aos 6 e 12 meses de idade, produzidas em biossólido (BIO) e substrato comercial (SC), sob diferentes doses de monoamônio fosfato amônio (MAP). $\mathrm{T}_{1}: \mathrm{BIO}+0,00 ; \mathrm{T}_{2}: \mathrm{BIO}+0,05 \mathrm{~g} \mathrm{de} \mathrm{MAP} \mathrm{muda}^{-1} ; \mathrm{T}_{3}: \mathrm{SC}+0,00 ; \mathrm{T}_{4}: \mathrm{SC}+0,05 \mathrm{~g} \mathrm{de} \mathrm{MAP} \mathrm{muda}^{-1} ; \mathrm{T}_{5}$ : $\mathrm{SC}+0,10 \mathrm{~g}$ de MAP muda ${ }^{-1} \mathrm{~T}_{6}: \mathrm{SC}+0,20 \mathrm{~g} \mathrm{de} \mathrm{MAP} \mathrm{muda}^{-1} ; \mathrm{T}_{7}: \mathrm{SC}+0,40 \mathrm{~g}_{\text {de MAP muda }}{ }^{-1}$. Para cada idade, colunas com a mesma letra não diferem entre si pelo teste de Tukey $(\mathrm{p}<0,05)$.

Figure 4. Height and diameter of Schinus terebinthifolia at 6 and 12 months old, produced in biosolid (BIO) and commercial substrate (SC), under different doses of monoammonium phosphate (MAP) . $\mathrm{T}_{1}: \mathrm{BIO}+0.00 ; \mathrm{T}_{2}: \mathrm{BIO}+$ $0.05 \mathrm{~g}$ of MAP seedlings ${ }^{-1} ; \mathrm{T}_{3}: \mathrm{SC}+0.00 ; \mathrm{T}_{4}: \mathrm{SC}+0.05 \mathrm{~g}$ of MAP seedlings $^{-1} ; \mathrm{T}_{5}: \mathrm{SC}+0.10 \mathrm{~g}$ of MAP seedlings ${ }^{-1}$; $\mathrm{T}_{6}: \mathrm{SC}+0.20 \mathrm{~g} \mathrm{MAP} \mathrm{seedlings}^{-1} ; \mathrm{T}_{7}: \mathrm{SC}+0.40 \mathrm{~g}$ of MAP seedlings $\mathrm{s}^{-1}$. For each age, columns with the same letter do not differ by Tukey test $(\mathrm{p}<0.05)$. 
implantação menores médias de altura, igualaram suas médias aos tratamentos $\mathrm{T}_{1}(\mathrm{BIO}+0,00)$ e $\mathrm{T}_{2}(\mathrm{BIO}+0,05)$ aos seis meses após a implantação e esta tendência se manteve após doze meses.

Segundo Salifu \& Timmer (2001), depois do plantio em campo o crescimento das raízes normalmente é lento e a absorção de nutrientes do solo é limitada. Dessa forma, o crescimento inicial depende da translocação de reservas internas de nutrientes. Este fato pode explicar o maior incremento e crescimento em altura do tratamento $\mathrm{T}_{7}$ (Figura 4), que pode ter sido favorecido pelo maior acúmulo de $\mathrm{N}$ e $\mathrm{P}$ dos tecidos, advindo da maior dosagem de MAP na adubação de cobertura.

A adoção de técnicas como a fertilização mineral pode trazer expressivos aumentos no crescimento e qualidade das mudas, com reflexos diretos na sobrevivência e crescimento em campo (Barbosa et al., 2003). Este mesmo comportamento também foi observado para o diâmetro do coleto. No entanto, para esta variável apenas o tratamento $\mathrm{T}_{7}(\mathrm{SC}+0,40)$ igualou-se às médias das mudas produzidas com biossólido (Figura 4).

Em plantios de recomposição florestal, onde diferentemente dos plantios comerciais de eucalipto as mudas nativas apresentam baixo ritmo de crescimento, é indispensável que sejam adotadas medidas que favoreçam o estabelecimento e crescimento mais rápido das mudas em campo, principalmente para diminuir a competição com as plantas daninhas. Pode-se inferir que tanto a adoção do biossólido como substrato, que resultou em mudas de maiores dimensões, como o uso de adubações de cobertura com doses mais elevadas de MAP, que aumentou o teor de nutrientes na planta e refletiu no melhor arranque inicial em campo, podem ser utilizados para favorecer o estabelecimento e crescimento em campo.

Considerando que atualmente existe uma grande tendência de verticalização das empresas que executam projetos de recomposição florestal, onde estas produzem as mudas florestais que utilizam em seus projetos, este trabalho traz resultados que podem contribuir para a tomada de decisão destes empreendedores. Em locais em que o biossólido seja de difícil obtenção, sugere-se que sejam testadas dosagens de fertilização em viveiro. Essa prática poderia ser utilizada para a formação de mudas com qualidade, uma vez que, possivelmente, uma dosagem maior de nutrientes na fase de viveiro, onde as condições ambientais são mais controladas, favoreceria o arranque inicial em campo.

\section{Conclusões}

A utilização do biossólido como substrato favoreceu o crescimento das mudas de aroeira pimenteira (Schinus terebinthifolia) na fase de viveiro, resultando em mudas de qualidade morfológica superior às das produzidas em substrato comercial com adubação de cobertura.

Tanto a utilização de biossólido como de substrato comercial, quanto o uso de adubação de cobertura com monoamônio fosfato em níveis mais elevados, podem ser considerados para acelerar o crescimento inicial das mudas de aroeira pimenteira em campo.

\section{Referências}

Abreu, A. H. M. et al. Produção de mudas e crescimento inicial em campo de Enterolobium Contortisiliquum produzidas em diferentes recipientes. Floresta, v. 45, n. 1, p. 141-150, 2015. DOI: 10.5380/ rf.v45i1.28931.

Abreu, A. H. M. et al. Urban solid waste in the production of Lafoensia pacari seedlings. Revista Brasileira de Engenharia Agrícola e Ambiental, v. 21, n. 2, p. 83-87, 2017. DOI: 10.1590/1807-1929/ agriambi.v21n2p83-87.

Barbosa, Z. et al. Crescimento e absorção de nutrientes por mudas de gravioleira. Revista Brasileira Fruticultura, v. 25, n. 3, p. 519-522, 2003. DOI: 10.1590/S0100-29452003000300039.

Basil, G. et al. Efecto del compost de biosólidos en la producción de plantines de Austrocedrus chilensis (ciprés de la cordillera). Ciencia del Suelo, n. 27, p. 49-55, 2009.

Birchler, T. et al. La planta ideal: revision del concepto, parametros definitorios e implementaction practica. Investigacion Agraria, Sistemas y Recursos Forestales, v. 7, n. 1, p. 109-121, 1998.

Brasil. Ministério do Meio Ambiente. Conselho Nacional do Meio Ambiente. Resolução CONAMA n 375/2006. Define critérios e procedimentos para o uso agrícola de lodos de esgoto gerados em estações de tratamento de esgoto sanitário e seus produtos derivados. Diário Oficial da República Federativa do Brasil, Brasília, DF, n. 167, p. 141-146, 30 ago 2006.

Brasil. Ministério de Minas e Energia. Departamento Nacional da Produção Mineral. Projeto RADAM Brasil: Folhas sc. 21. Juremo: geomorfologia, pedologias, vegetação e uso potencial da terra. Rio de Janeiro, 1980. 420 p.

Cabreira, G. V. et al. Biossólido como componente de substrato para produção de mudas florestais. Floresta, v. 47, n. 2, p. 165 - 176, 2017. DOI: $10.5380 /$ rf.v47i2.44291.

Caldeira, M. V. W. et al. Biossólido na composição de substratos para produção de mudas de Tectona grandis. Floresta, v. 42, n. 1, p. 77-84, 2012. DOI: 10.5380/rf.v42i1.26302.

Caldeira, M. V. W. et al. Composto orgânico na produção de mudas de aroeira-vermelha. Scientia Agraria, v. 9, n. 1, p. 27-33, 2008. DOI: $10.5380 /$ rsa.v9i1.9898. 
Caldeira, M. V. W. et al. Lodo de esgoto e vermiculita na produção de mudas de eucalipto. Pesquisa Agropecuária Tropical, v. 43, n. 2, p. 155-163, 2013a.

Caldeira, M. V. W. et al. Substratos alternativos na produção de mudas de Chamaecrista desvauxii. Revista Árvore, v. 37, n. 1, p. 31-39, 2013b. DOI: 10.1590/S0100-67622013000100004.

Carneiro, J. G. A. Produção e controle de qualidade de mudas florestais. Campos: UNEF, 1995, 451 p.

Carvalho, P. E. R. Espécies arbóreas brasileiras. Colombo: Embrapa Florestas, 2003. 1039 p.

Delarmelina, W. M. et al. Uso de lodo de esgoto e resíduos orgânicos no crescimento de mudas de Sesbania virgata (Cav.) Revista Agroambiente on-line, v. 7, n. 2, p. 184-192, 2013. DOI: 10.18227/1982-8470ragro.v7i2.888.

Dickson, A. et al. Quality appraisal of white spruce and white pine seedling stock in nurseries. Forestry Chronicle, v. 36, p. 10-13, 1960. DOI: $10.5558 / \mathrm{tfc} 36010-1$.

Instituto Nacional de Meteorologia. Precipitação diária e temperatura máxima Cordeiro, RJ. Disponível em: $<$ http://www. inmet.gov.br/html/observacoes.php?>. Acesso em: 15 mar. 2014.

Johnson, J. D. \& Cline, M. L. Seedling quality of southern pines. In: Dureya, M. L. \& Dougherty, P. M. (Ed.). Forest regeneration manual. Dordrecht: Kluwer Academic Publishers, 1991. p. 143-162.

José, A. C. et al. Produção de mudas de aroeira (Schinus terebynthifolius Raddi) para recuperação de áreas degradadas pela mineração de bauxita. Cerne, v. 11, n. 2, p. 187-196, 2005.

Lorenzi, H. Árvores brasileiras: manual de identificação e cultivo de plantas arbóreas nativas do Brasil. 2. ed. Nova Odessa: Platarum, 2002. v. 2.

Martins, S. V. Recuperação de matas ciliares. Viçosa, MG: Aprenda Fácil, 2001. 75 p.
Melo, L. A. et al. Qualidade e crescimento inicial de mudas de Mimosa caesalpiniifolia benth.produzidas em diferentes volumes de recipientes. Ciência Florestal, v. 28, n. 1, p. 47-55, 2018. DOI: 10.5902/1980509831574.

Namiot G. et al. Producción de plantines de arbóreas nativas. Experiencias con ciprés de la cordillera. In: Mazzarino, M. J. \& Satti, P. (Ed.). Compostaje en la Argentina: experiencias de producción, calidad y uso. Buenos Aires: Orientación, 2012. 349 p.

Nóbrega, R. S. A. et al. Utilização de biossólido no crescimento inicial de mudas de aroeira (Schinus terebynthifolius Raddi). Revista Árvore, v. 31, n. 2, p. 239-246, 2007.

Pezzutti, R. V. \& Caldato, S. L. Sobrevivência e crescimento inicial de mudas de Pinus taeda L. com diferentes diâmetros do colo. Ciência Florestal, v. 21, n. 2, 2011. DOI: 10.5902/198050983240.

Ribeiro Júnior, J. I. Análises estatísticas no SAEG. Viçosa, MG: UFV, 2001. 301 p.

Salifu, K. F. \& Timmer, V. R. Nutrient retranslocation response of Picea mariana seedlings to nitrogen supply. Soil Science Society of America Journal, n. 65, p. 905-913, 2001. DOI: 10.2136/ sssaj2001.653905x.

Santos, F. E. V. et al. Características químicas de substratos formulados com lodo de esgoto par produção de mudas florestais. Revista Brasileira de Engenharia Agrícola e Ambiental, v. 18, n. 9, p. 971-979, 2014. DOI: 10.1590/1807-1929/agriambi. v18n09p971-979.

Trazzi, P. A. et al. Produção de mudas de Tectona grandis em substratos formulados com biossólido. Cerne, v. 20, n. 2, p. 293-302, 2014. DOI: 10.1590/01047760.201420021134.

Trigueiro, R. M. \& Guerrini, I. A. Utilização de lodo de esgoto na produção de mudas de Aroeira pimenteira. Revista Árvore, v. 38, n. 4, p. 657-665, 2014. DOI: 10.1590/S0100-67622014000400009.

Varela, S. \& Martinez, A. Uso del compost de biossólidos em la formulación de sustratos para la producción industrial de plantas de Nothofagus alpina. Bosque, v. 34, n. 3, p. 281-289, 2013. 